\title{
Role of CT Guided Celiac Plexus Block for Intractable Abdominal Pain
}

Amr Mahmoud Zayed, Mahmoud Ibrahim Elshamy, Amr Alaa Ramadan El sakka *

Department of Radiodiagnosis, Faculty of Medicine - Al-Azhar University

*Correspondence author: Amr Alaa Ramadan El sakka; Mobile: (+20) 01116107784; E-mail: amr_elsakka_2010@yahoo.com

\begin{abstract}
Background: Over the last decades, the incidence of pancreatic cancer has increased. It is the seventh most common cause of cancer deaths especially in the developed world.

Objective: This study aims to evaluate the efficacy of computed tomography (CT) guided celiac plexus neurolysis (CPN) to relieve intractable abdominal pain caused mainly by abdominal malignancy.

Patients and Methods: This prospective study included 20 adult patients; some of them were referred from Oncology Department and others were from outpatient clinic. Patients had abdominal pain owing to abdominal cancer during the period from October 2018 to May 2019 in Al-Hussien and Bab-Alsheria Hospitals, Al- Azhar University.

Results: CT-guided celiac plexus block was successfully carried out for the patients. 13 of them (65\%) were males and $7(35 \%)$ were females, with mean age of 57.05 years. Abdominal pain in the 20 patients was owing to infiltrative or metastatic hepatocellular carcinoma in ten $(50 \%)$ patients, pancreatic cancer in six (30\%) patients, lymphoma in two $(10 \%)$ patients, gastric cancer in one $(5 \%)$ patient, and metastatic lung in cancer one $(5 \%)$ patient.

Conclusion: The current study disclosed the incremental value of the CT-guided CPN via using different techniques with injection of $20-40 \mathrm{~mL}$ of $95 \%$ of ethanol neurolytic solution as an ideal palliative treatment for eradication of severe pain mainly caused by unresectable pancreatic cancer.
\end{abstract}

Keywords: CT, celiac plexus block, intractable abdominal pain

\section{INTRODUCTION}

Celiac plexus block was used in various upper abdominal malignant and non-malignant pain syndromes with variable success. Pain signals stemming from visceral structures that are innervated by the celiac plexus can be interrupted by blocking the celiac plexus or the splanchnic nerves. These structures include the pancreas, liver, gallbladder, mesentery, omentum, and gastrointestinal tract from the lower esophagus to the transverse colon $^{(1)}$.

The most common application of neurolytic celiac plexus block is upper abdominal malignancy, especially pancreatic cancer; this was first described by Kappis in $1914^{(2)}$.

Cancer-related pain remains a common problem in oncologic practice and has a major influence on patient's comfort, tolerance of therapies, and probably survival. Timely interventional cancer pain therapies complement conventional pain management by reducing the need for high-dose opioid therapy and the associated toxicity ${ }^{(3)}$.

Abdominal viscera including pancreas, liver, gallbladder, adrenal, kidneys, and the gastrointestinal tract from the level of the gastroesophageal junction to the splenic flexure of the transverse colon are innervated by the celiac plexus. So, pain owing to tumors in these viscera may be relieved through the use of a neurolytic celiac plexus block ${ }^{(4,5)}$.

\section{AIM OF THE WORK}

This study aims to evaluate the efficacy of computed tomography (CT) guided celiac plexus neurolysis to relieve intractable abdominal pain caused mainly by abdominal malignancy.

\section{PATIENTS AND METHODS}

This prospective study was carried out on 20 adult patients referred from Oncology Department having abdominal pain owing to abdominal cancer during the period from October 2018 to May 2019 in Al-Hussien and Bab-Alsheria Hospitals, Al-Azhar University.

\section{Ethical approval}

The study protocol was approved by the Medical Ethical Committee of Faculty of Medicine, Al-Azhar University.

After an informed consent was taken from each patient included in the study, all the patients underwent CT-guided CPN with unilateral or bilateral paramedian needle entry using anterior and posterior approach techniques.

\section{Inclusion criteria}

The inclusion criteria were abdominal pain due to abdominal cancer, pain not controlled by analgesics, and patients having adverse effects of analgesic drugs.

\section{Exclusion criteria}

The exclusion criteria were patient's refusal of the procedure, patients with coagulopathy, and patients with marked ascites.

\section{Method}

Patient preparation: All the patients included were subjected to detailed medical history, general examination, and other investigations such as abdominal ultrasound, CT abdomen, and coagulation profile. The patients were asked to stop analgesics over the night before the procedure, fast for $8 \mathrm{~h}$, and were informed about the complications and hospital stay time 
(usually a night before the procedure for preparation and $4 \mathrm{~h}$ after under observation). Equipment and drugs: multiple syringes $(3,5,10$ and $20 \mathrm{ml})$, sterile dressing, betadine and alcohol for sterilization, multislice widebore gantry SOMATOM Definition Flash CT Scanners 4 and 16 slices (Siemens Healthineers Global, Erlangen, Germany), lidocaine 2\%, ethyl alcohol 20-40 $\mathrm{ml} \mathrm{95 \% ,}$ Chiba needle 20 or $22 \mathrm{G}$ with length $20 \mathrm{~cm}$, intravenous cannula (18 G), intravenous, fluids and resuscitation drugs (ephedrine, atropine) were used during the procedure.

\section{Procedure:}

Intravenous cannula size $18 \mathrm{G}$ was inserted. A control axial scanning of the abdomen was taken to localize the region of interest, which covers the celiac plexus location and the course of the needle to the celiac plexus.

After sterilization of the skin, the local cutaneous and subcutaneous anesthetic (lidocaine 2\%) was injected. Then a $20-\mathrm{cm}$ long $20-\mathrm{G}$ or $22-\mathrm{G}$ Chiba needle was introduced in paramedian plane. Using serial localized CT scans focused on the region of interest, the needle was advanced into the right lateral or the left lateral area to the celiac trunk origin.

Once the tip of the needle confirmed to be correctly positioned, suction was applied to confirm that the needle tip was not inside a blood vessel, then a 'prognostic block' was performed by injection of a local anesthetic (12 $\mathrm{ml}$ of lidocaine $2 \%$ ) mixed with $2 \mathrm{ml}$ of nonionic contrast material (Ultravist; Bayer HealthCare Pharmaceuticals Inc., Wayne, Germany) to show the distribution of the lidocaine under the CT images. Injection of local anesthetic should always precede that of ethanol to reduce the pain from ganglion neurolysis and also for enforcement of a diagnostic celiac plexus block.

After 5-10 min if the injection successfully relieved pain (successful block), 20-40 $\mathrm{ml}$ of $95 \%$ ethanol mixed with 3-ml contrast was used to show the distribution of the ethanol. Before the needle was removed, $3 \mathrm{ml}$ of lidocaine $2 \%$ was injected to relieve the irritation caused by ethanol. Immediately after needle withdrawal, serial CT scans were obtained to cover the area from the upper border of the $12^{\text {th }}$ thoracic to the lower border of the first lumbar vertebrae to visualize the spread of the neurolytic solution.

The patients had to stay in the hospital for $4 \mathrm{~h}$ under observation. Patients were evaluated using the visual analog scale (VAS) score for assessment of the degree of pain relief immediately after injection and 1 week, 1 month, and 3 months after neurolysis. Moreover, analgesic requirements and complications were documented. A decrease in the VAS score to $50 \%$ or more of the baseline value was considered significant.

\section{Statistical methods}

Data were coded and entered using the statistical package SPSS (Statistical Package for the Social Sciences) version 25. Data was summarized using mean, standard deviation, median, minimum and maximum in quantitative data and using frequency (count) and relative frequency (percentage) for categorical data. 


\section{RESULTS}

CT-guided celiac plexus block was carried out for 20 adult patients.

The technique was successfully performed in $20(100 \%)$ patients, 13 of them $(65 \%)$ were males and $7(35 \%)$ were females, with mean age of 57.05 years. Abdominal pain in the 20 patients was owing to infiltrative or metastatic hepatocellular carcinoma in ten (50\%) patients [Figure 1], pancreatic cancer in six (30\%) patients, lymphoma in two (10\%) patients, gastric cancer in one (5\%) patient, and metastatic lung cancer one (5\%) patient [Figure 2].
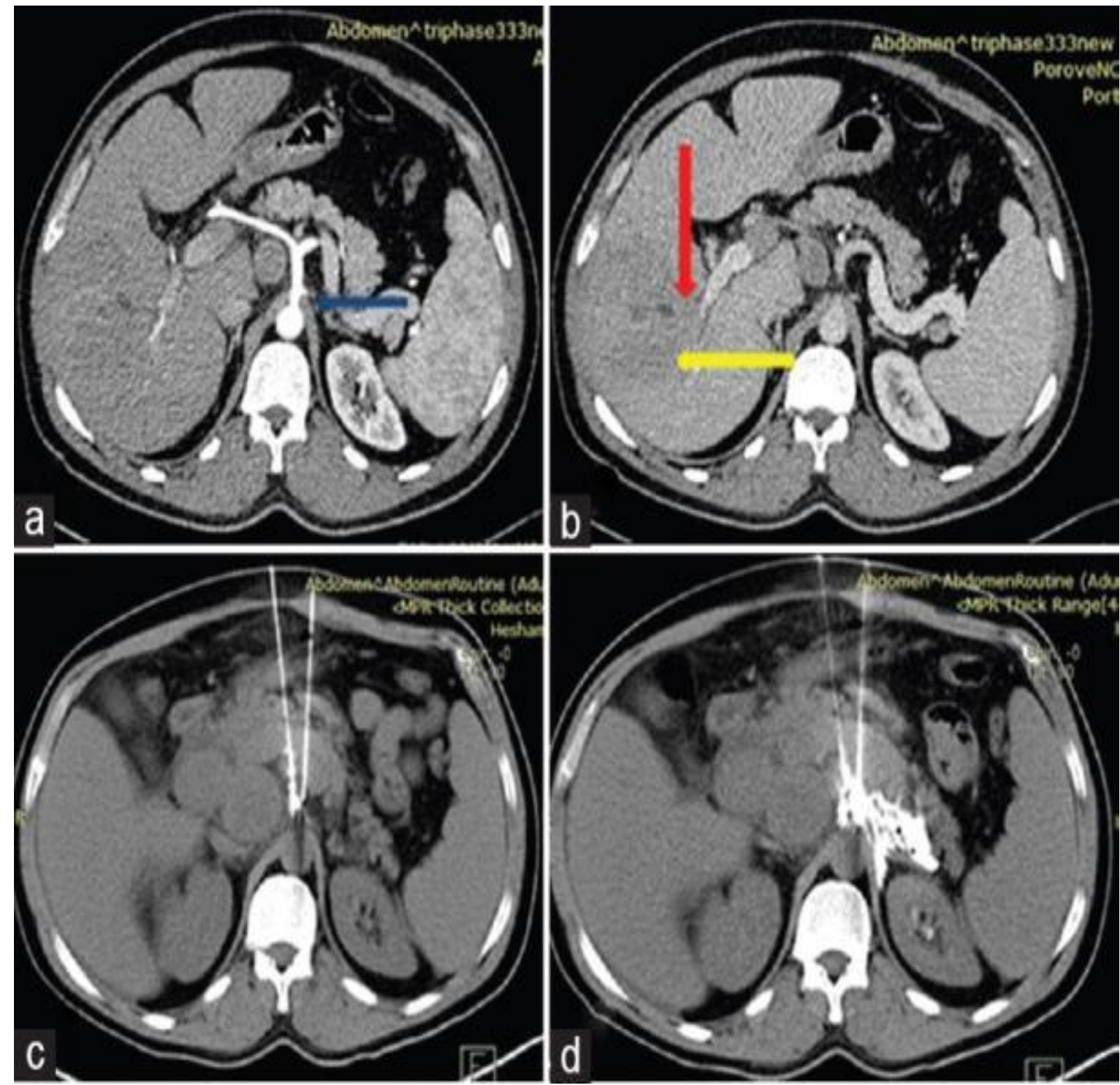

Figure 1: A 42-year-old patient with hepatocellular carcinoma invading the right portal vein undergoing bilateral celiac plexus neurolysis (CPN) procedure. (a, b) Axial computed tomography images show the celiac artery origin in arterial phase (blue arrow), the hepatic focal lesion (yellow arrow), and the right portal vein invasion in portal phase (red arrow). (c, d) Axial computed tomography images of bilateral CPN procedure show the two Chiba needles at both celiac plexuses before ethanol injection (c) and after (d). Before CPN, pain degree by visual analog scale score was: 10/10. Then after CPN, it became immediately 2/10, 1 week after CPN 1/10, 1 month after CPN 1/10, and 3 months later $1 / 10$. 

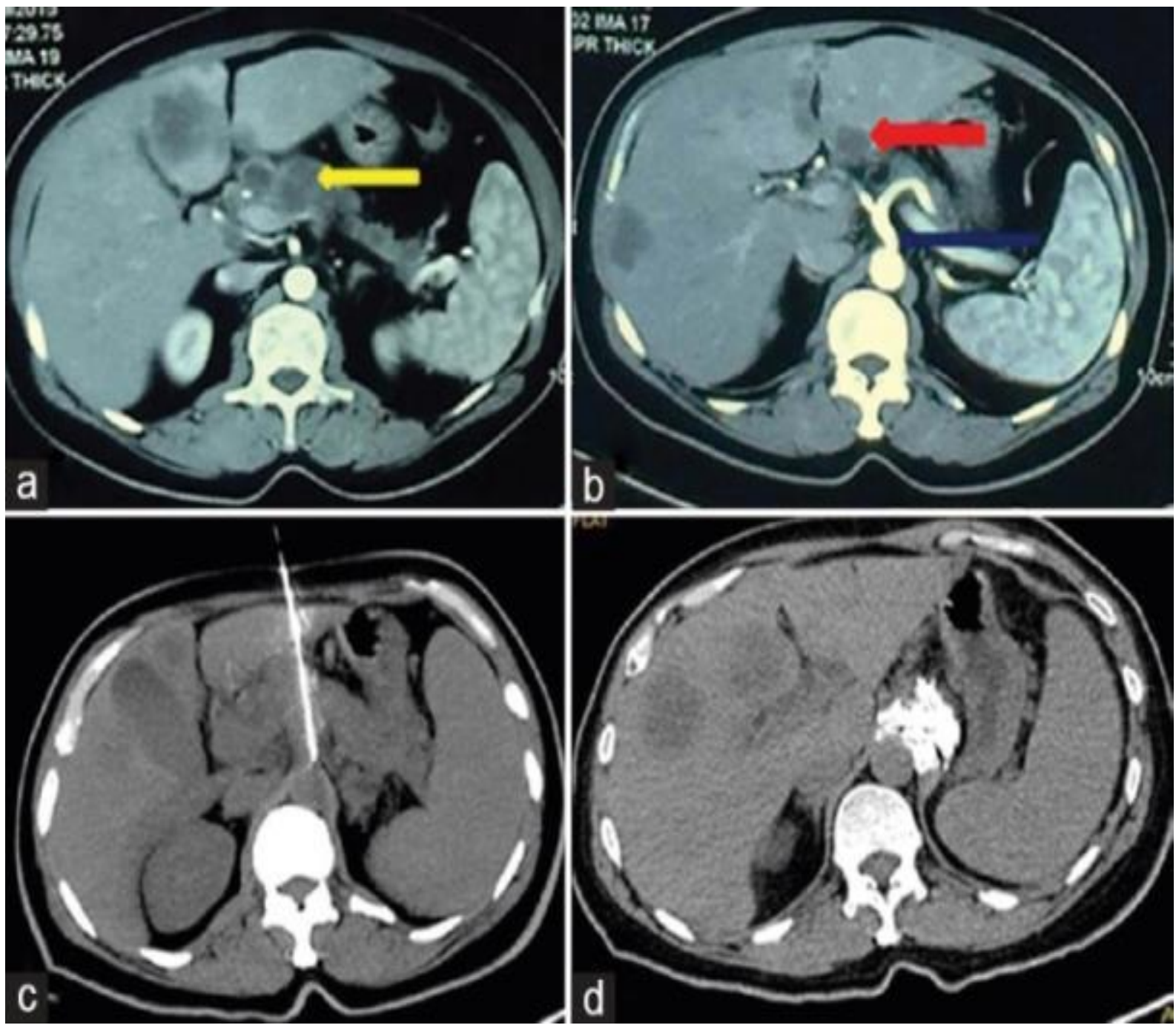

Figure 2: A 52-year-old patient with metastatic lung cancer to the liver and paraaortic lymph nodes undergoing unilateral paramedian celiac plexus neurolysis (CPN) procedure. (a, b) Axial computed tomography images show nodal metastasis (yellow arrow), liver deposits (red arrow), and celiac trunk (blue arrow). (c, d) Axial computed tomography images of unilateral CPN procedure show the course of the needle with its tip at the left celiac plexus (c), whereas (d) shows the distribution of ethanol after needle removal. Before CPN, pain degree by visual analog scale score was 9/10. Then immediately after CPN, it was 2/10; 1 week after CPN was 1/10; 1 month after CPN was 2/10; and 3 months later was $2 / 10$.

Table (1): Distribution age and sex.

\begin{tabular}{|c|c|c|c|c|c|}
\hline & Mean & Standard Deviation & Median & Minimum & aximum \\
\hline Age & 57.05 & 6.75 & 58.00 & 45.00 & 66.00 \\
\hline Pre Pain degree & 8.60 & 0.50 & 9.00 & 8.00 & 9.00 \\
\hline Follow up pain degree & 0.75 & 0.72 & 1.00 & 0.00 & 2.00 \\
\hline Death duration (months) & 3.12 & 1.86 & 3.00 & 0.50 & 6.00 \\
\hline & & \multicolumn{2}{|l|}{$\overline{\text { Count }}$} & \multicolumn{2}{|l|}{$\%$} \\
\hline \multirow{2}{*}{ Sex } & \multirow{2}{*}{$\frac{\mathbf{M}}{\mathbf{F}}$} & \multicolumn{2}{|l|}{13} & \multicolumn{2}{|l|}{$65.0 \%$} \\
\hline & & \multicolumn{2}{|l|}{7} & \multicolumn{2}{|l|}{$35.0 \%$} \\
\hline \multirow{2}{*}{ Death } & Yes & \multirow{2}{*}{\multicolumn{2}{|c|}{$\frac{17}{3}$}} & \multicolumn{2}{|l|}{$85.0 \%$} \\
\hline & No & & & \multicolumn{2}{|l|}{$15.0 \%$} \\
\hline
\end{tabular}


CASE (1)

\section{Illustrative Cases}
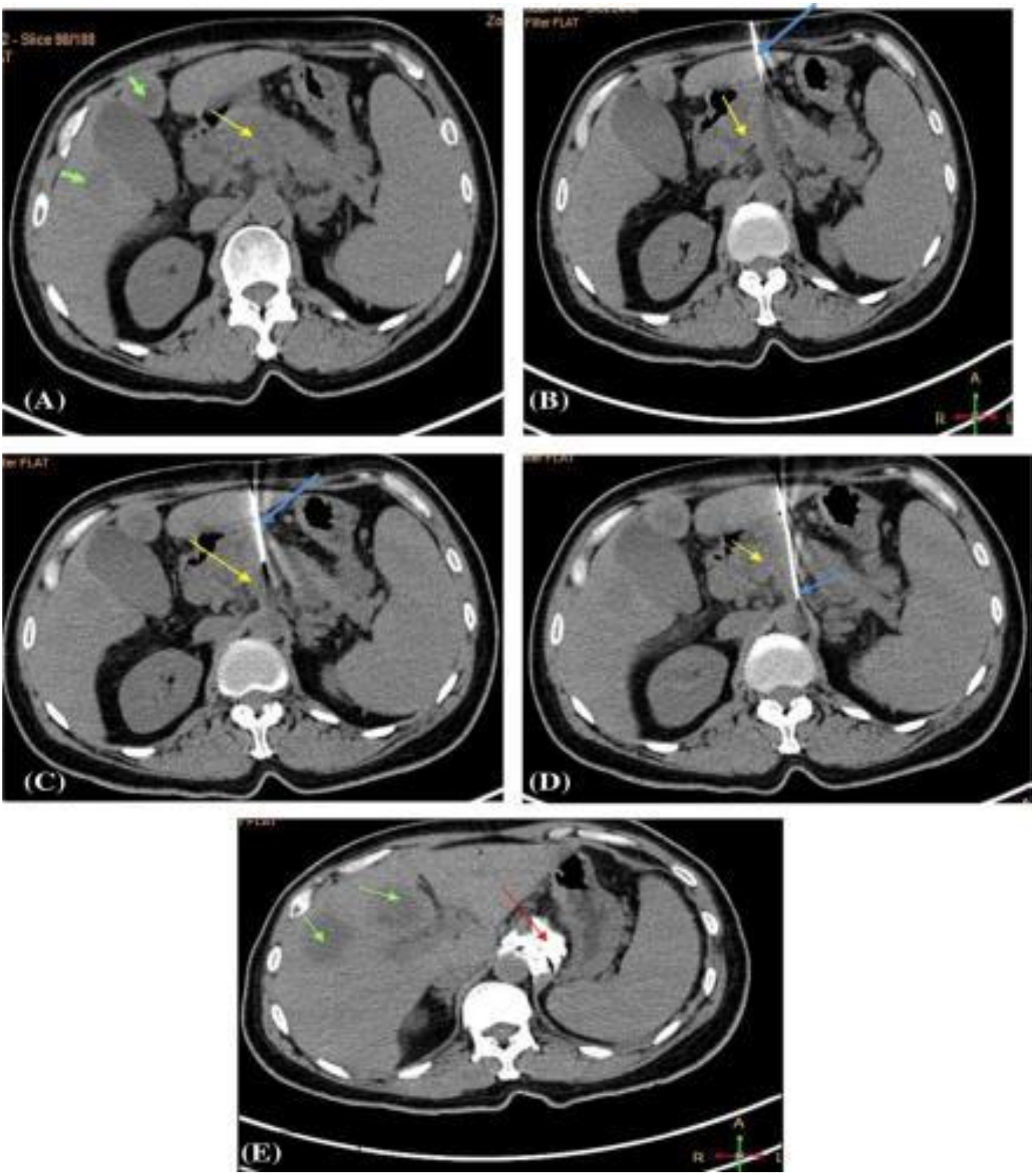

Figure 3: Axial abdominal CT images of 62 year old male patient with adenocarcinoma of pancreatic body, invading the celiac plexus (yellow arrows) and complicated with liver metastases (green arrows). Before CPN, the VAS score of pain of this patient was 9/10. The CT-guided CPN was successfully achieved by using a single puncture technique, where the puncture needle was successfully introduced via median anterior approach with the needle tip was seen at the level of celiac axis (blue arrows).

The CT images, obtained after completion of CPN and injection of a mixture of $5 \mathrm{ml}$ contrast media and $25 \mathrm{ml}$ of 95\% ethanol as a neurolytic agent, show adequate spread of the neurolytic agent that appears as bright hyper-dense area on CT images, around entire celiac axis (red arrows). The VAS score of pain of this patient became 2/10 immediately after the procedure, $1 / 10$ one week after CPN and 2/10 three months after CPN. 
CASE (2)
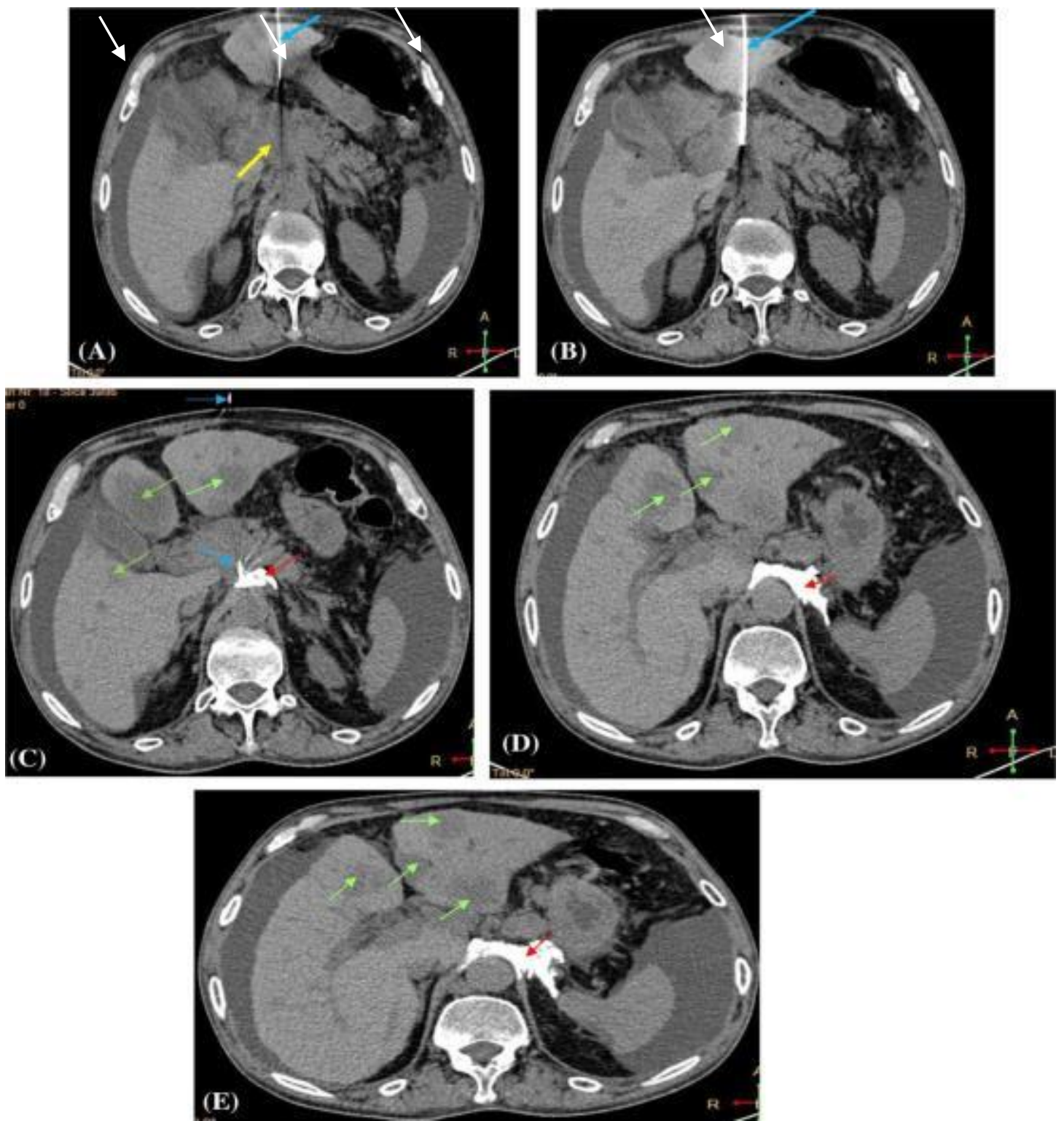

Figure 4: Axial abdominal CT images of 69 year old male patient with pancreatic cancer, invading the celiac plexus (yellow arrow) and complicated with liver metastases (green arrows) and ascites. Before CPN, the VAS score of pain of this patient was 8/10. The CT-guided CPN was successfully achieved by using a single puncture technique, where the puncture needle was successfully introduced vertically via median anterior approach with the needle tip was seen at the level of celiac axis (blue arrows).

The CT images, obtained after injection of a mixture of $5 \mathrm{ml}$ contrast media and $30 \mathrm{ml}$ of $95 \%$ ethanol (the neurolytic agent), demonstrate the adequate spread of the neurolytic agent, which appears as bright hyper-dense area, around entire celiac axis (red arrows). The VAS score of pain of this patient became 2/10 immediately after CPN, 1/10 one week after CPN and 1/10 three months after CPN. 


\section{CASE (3)}
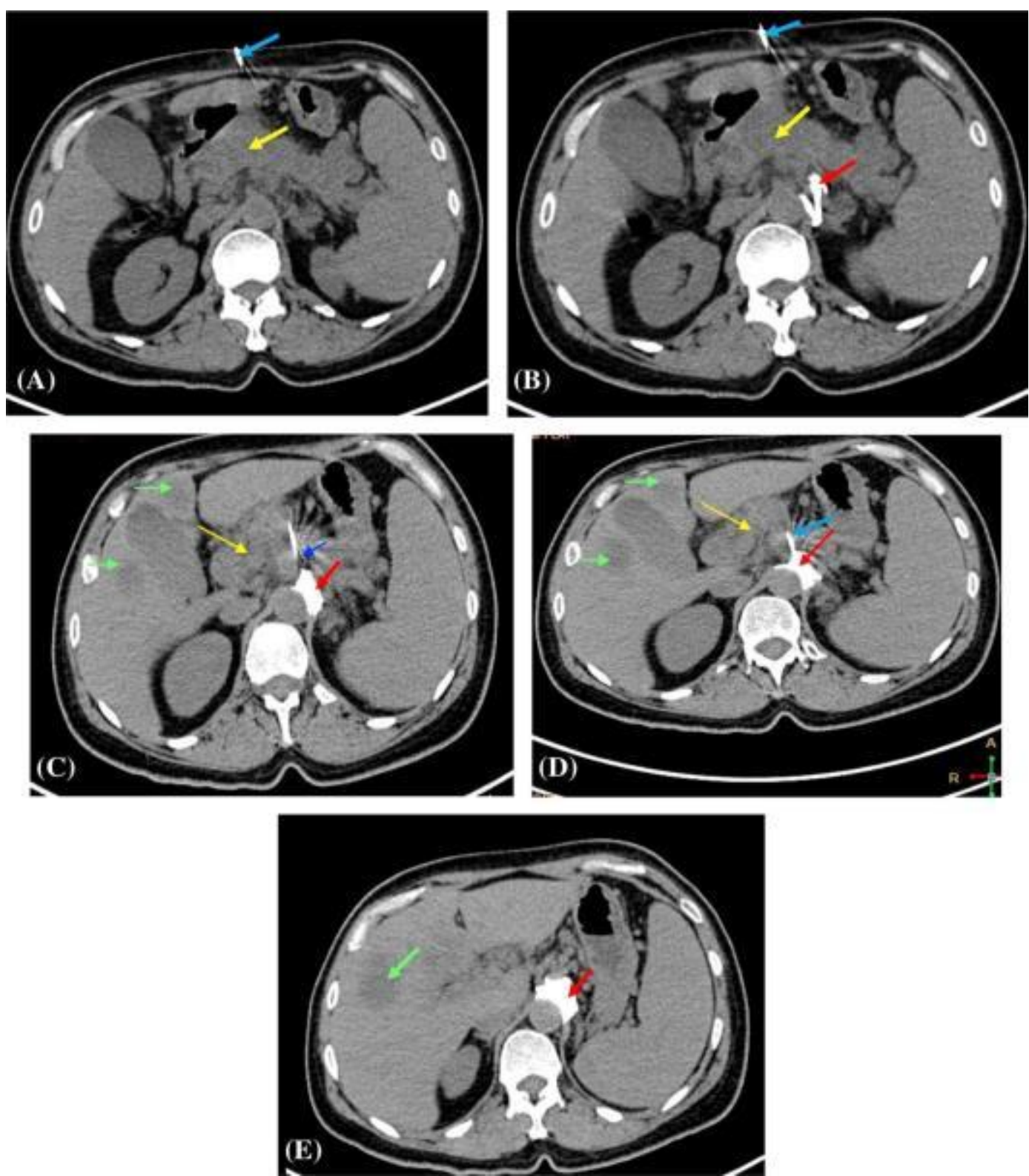

Figure 5: Axial abdominal CT images of 56 year old female patient with pancreatic adenocarcinoma (yellow arrows) complicated with liver metastases (green arrows). Before CPN, the VAS score of pain of this patient was 7/10. The CT-guided CPN was successfully achieved by using a single puncture technique, where the puncture needle was successfully introduced via median anterior approach with the needle tip was seen at the level of celiac axis (blue arrows). The CT images, obtained after injection of a mixture of $5 \mathrm{ml}$ contrast media and $25 \mathrm{ml}$ of $95 \%$ ethanol (the neurolytic agent) show adequate spread of the neurolytic agent, which appears as bright hyper-dense area on CT images, around celiac axis and left celiac ganglia (red arrows). The VAS score of pain of this patient became 2/10 immediately after CPN, 1/10 one week after CPN and 1/10 three months after CPN. 

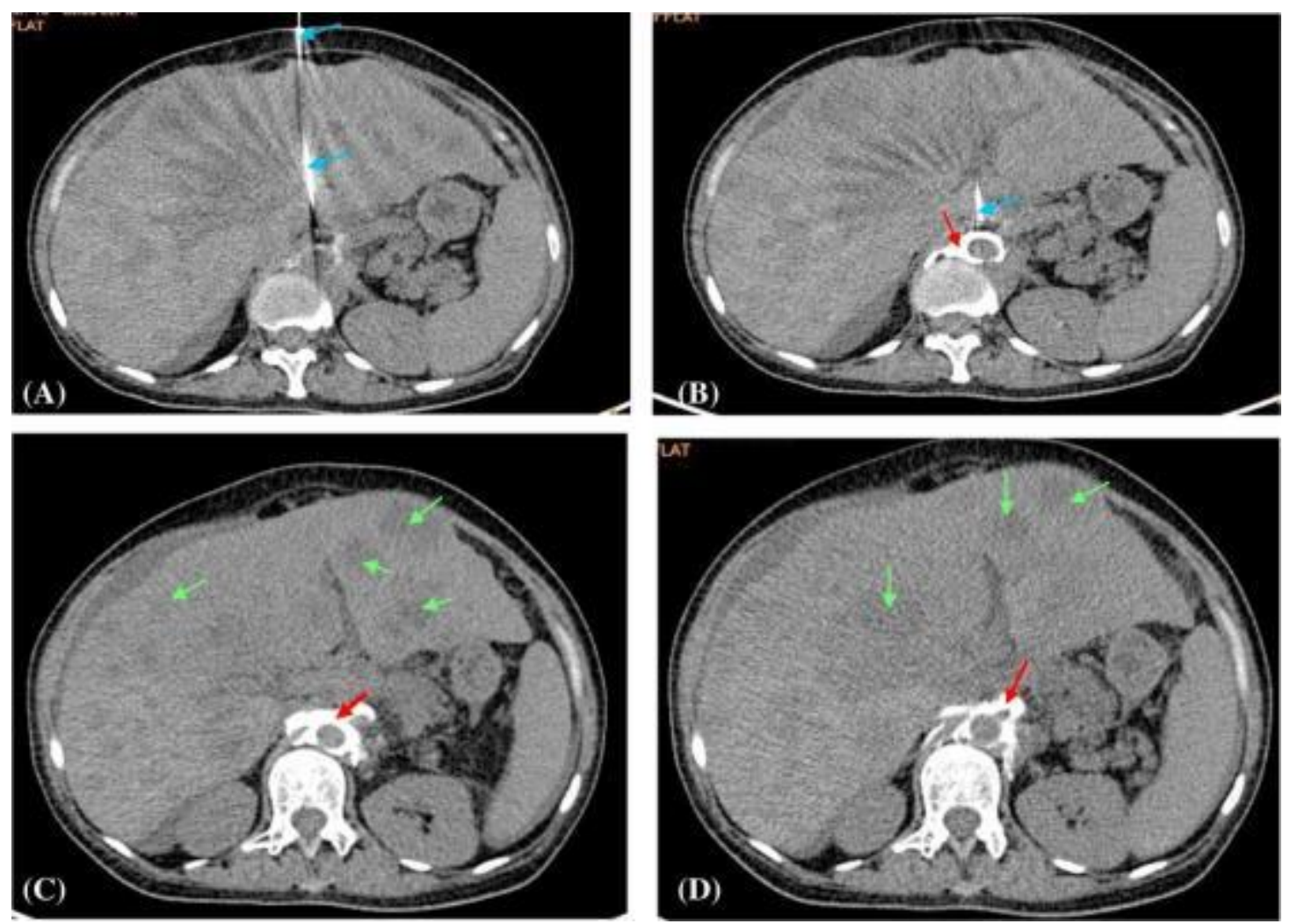

Figure 6: Axial abdominal CT images of 58 year old female patient with adenocarcinoma of pancreatic cancer complicated with liver metastases (green arrows) and ascites. Before CPN, the VAS score of pain of this patient was 9/10. The CT-guided CPN was successfully achieved by using a single puncture technique, where the puncture needle was successfully introduced vertically via median anterior approach with the needle tip was seen at the level of celiac axis (blue arrows). The CT images, obtained after injection of a mixture of $5 \mathrm{ml}$ contrast media and $25 \mathrm{ml}$ of $95 \%$ ethanol (the neurolytic agent), demonstrate a bright hyper-dense area of neurolytic agent on CT images with adequate spread of the neurolytic agent around the entire celiac axis (red arrows). The VAS score of pain of this patient became 2/10 immediately after CPN, 1/10 one week after CPN and 1/10 three months after CPN.

\section{DISCUSSION}

The CPN is an adjunctive form of palliative pain management. It is performed by different techniques, such as anterior approach, posterior approach and percutaneous CPN . Also, the CPN may be guided by different modalities such as fluoroscopy, tomography, and endoscopic ultrasound-guidance. The CT-guidance is replacing other techniques because it allows direct visualization of neurolytic agent diffusion in the retroperitoneal anatomic space with correct needle positioning. Additionally, it permits 3-dimensional information of the position of the celiac artery and direct visualization of anatomic structures, such as pancreas, and important vascular structures, particularly the aorta, celiac trunk, and superior mesenteric artery, preventing their injuries ${ }^{(6)}$.

In our study, we included twenty patients, who were suffering from severe intractable abdominal pain mainly due to unresectable pancreatic cancer in order to perform $\mathrm{CPN}$ as a palliative management of such pain. CPN is recommended through the posterior approach. However, in patients with disturbed anatomy, the posterior approach for CPN cannot be used. Moreover, the posterior approach leaves the patient in the prone position for a long time, which can be distressing for patients with intra-abdominal pain. On the other side, the anterior approach lets the patients to be in comfortable supine position throughout the procedure. Additionally, the posterior approach is more vulnerable to neurological complications . On the other hand, the anterior approach carries a reduced risk of neurologic complications since needle tip is anterior to the spinal arteries and spinal canal. We used the anterior approach in most cases to perform CPN as it is easier, safe and more comfortable than the posterior approach especially in advanced disease. Also, the anterior approach makes the needle not impinging on either periosteum, nerve roots or pass through the paraspinous musculature with less risk of accidental neurologic injury and abolishing the potential risk of paraplegia ${ }^{(6)}$.

The methods used to place the needle and obtain the best response of CPN, are still controversial. These methods include the single (unilateral or median) and the bilateral para-median on both sides of celiac trunk. The choice between the single or bilateral methods remains 
difficult, depending on the personal skills and the experience of every operator ${ }^{(7)}$.

In all of our patients, we successfully achieved CPN via using anterior and posterior approaches. anterior approach was preferred due to using the single puncture method in order to reduce the patients' distress and decrease the amount of the used neurolytic agent, with avoidance of the risk of puncturing the aorta. However, the bilateral paramedian needle entry technique, despite its more adverse events, due to greater needle movement. They thought that the technique of single puncture was not adequately exposing the celiac ganglia to ethanol. (The 2 paragraphs in blue have similar repeated meaning. Rewrite them in 1 paragraph after deleting the repetition)

In order to interrupt neural networks of the celiac plexus, chemical ablative procedures are preferred to other mechanical modalities. The commonly exploited neurolytic agents in CPN were alcohol in concentrations of $50-100 \%$. The mechanism of alcohol neurolysis involves extraction of cholesterol/phospholipid and cerebroside from the neural membranes and by precipitation of mucoproteins. Neurolytic action of phenol is exerted by virtue of causing protein coagulation and necrosis when directly applied to the nerves . Ethanol is relatively safe and a good agent for relieving malignancies-related pain with a longer duration of pain control and a significant reduction in opioid requirements ${ }^{(\boldsymbol{8})}$.

In the present study, all of the studied patients had effective immediate pain relief with VAS scores of pain markedly reduced to less than 3 . We observed highly significant $(P<0.001)$ distinction in the VAS scores of pain in the studied patients before and after CPN. The peak reduction of the pain sensation was observed in the first day after the CPN.

A good pain relief response was observed in patients, who consumed some NSAIDs after the procedure but in small doses.

The CPN is a safe and cost-effective approach to treat visceral pain associated with cancer. Complications associated with CPN are considered to be due to interruptions of the autonomic system caused by ablation of the celiac plexus and sympathetic blockade leading to unopposed parasympathetic activity. We found the most common complications after CPN were; transient irritant pain after $\mathrm{CPN}$ at the puncture site, transient diarrhea, nausea, vomiting and post-procedural postural hypotension, which effectively responded to intravenous fluid therapy ${ }^{(9)}$.

The local abdominal and back pain, during or immediately after CPN, were commonly encountered as a result of the ablative effect of the neurolytic agent.
Also, the common transient post-procedural complications such as diarrhea, which resolved in around $48 \mathrm{~h}$, are related to the sympathetic blockade and unopposed parasympathetic efferent influence after the block. Moreover, the post-procedural orthostatic hypotension can be explained by loss of sympathetic tone and dilated abdominal vasculature ${ }^{(\mathbf{1 0})}$.

\section{Conclusion}

The current study disclosed the incremental value of the CT-guided CPN via using deferent techniques with injection of $20-40 \mathrm{ml}$ of $95 \%$ of ethanol neurolytic solution as an ideal palliative treatment for eradication of severe pain mainly caused by unresectable pancreatic cancer.

Our study revealed that in a seriously impaired pancreatic cancer patient scenario, CPN appears as a safe minimally invasive technique, which is easy to perform, well accepted and tolerated. It is effective in pain relief up to $100 \%$ of the studied patients . After CPN, patients showed an excellent response to the procedure with excellent pain relief . Also, there was a significant reduction $(P<0.001)$ in the analgesic requirements after CT-guided CPN.

\section{REFERENCES}

1. Wong GY, Schroeder DR, Carns PE et al. (2004): Effect of neurolytic celiac plexus block on pain relief, quality of life, and survival in patients with unresectable pancreatic cancer: A randomized controlled trial. JAMA., 291:1092-1099.

2. Kappis M (1914): Erfahrungen mit lokalanasthesie beibauchoperationen. Verh Dtsch Ges Chir., 43:87-89.

3. Rana MV, Candido KD, Raja O et al. (2014): Celiac plexus block in the management of chronic abdominal pain. Curr Pain Headache Rep., 18:394-398.

4. Polati E, Luzzani A, Schweiger V et al. (2008): The role of neurolytic celiac plexus block in the treatment of pancreatic cancer pain. Transplant Proc., 40:1200-1204.

5. Arai YP, Morimoto A and Sakurai H (2013): The effect of celiac plexus block on heart rate variability. J Anesth., 2013; 27:62-65.

6. Jain P, Dutta A, Sood J (2006): Coeliac plexus blockade and neurolysis: an overview. Indian J Anaesth., 50(3):169-77.

7. Eisenberg E, Carr DB, Chalmers TC (1995): Neurolytic celiac plexus block for treatment of cancer pain: a meta-analysis. Anesth Analg., 80(2):290 295.

8. McGuire $\mathrm{D}$, Kaiser $\mathrm{K}$, Haisfield-Wolfe $\mathrm{M}$ et al. (2016): Pain Assessment in Non-Communicative Adult Palliative Care Patients. Nursing Clinics of North America, 51(3):397-431.

9. Moore DC (2004): The dreaded complications from neurolytic celiac plexus blocks are preventable! Reg Anesth Pain Med., 29(4):377-378.

10.Soweid AM, Azar C (2010): Endoscopic ultrasoundguided celiac plexus neurolysis. World J Gastrointest Endosc., 2(6):228-231. 